\title{
Tratamiento de la brucelosis humana
}

\author{
Treatment of human brucellosis
}

Skalsky K, BMJ 2008; 336(7646):701-4.

\section{Objetivo}

Determinar la eficacia de distintos tratamientos de la brucelosis.

Fuente de datos, selección de estudios y extracción de datos PUBMED CENTRAL, LILACS y congresos sin restricción de lenguaje; estudios no publicados, listas de referencias y registros de ensayos en curso; pesquisando ensayos controlados y aleatorizados o cuasi- aleatorizados* que hubieran incluido monoterapia antibiótica vs. terapia combinada, en comparación con placebo/no tratamiento u otra terapia antibiótica; al igual que diferente duración del tratamiento; con infección documentada clínica y serológicamente. El resultado primario fue la recaída clínica o serológica y la falla terapéutica; y los secundarios, la aparición de complicaciones, enfermedad no presente inicialmente y/o efectos indeseables, la discontinuación del tratamiento y la mortalidad global.

\section{Resultados principales}

Se incluyeron 30 de 110 estudios seleccionados que habían evaluaron 77 esquemas terapéuticos. Ver tabla 1.

Tabla 1: eficacia de los diferentes tratamientos de brucelosis.

\begin{tabular}{|c|c|c|c|}
\hline Intervención vs. Comparación & Falla globalRR (IC95\%) & Abandono RR (IC95\%) & Efectos adversosRR (IC95\%) \\
\hline $\begin{array}{l}\text { Tetraciclina-Estreptomicina (TE) vs. Tetraciclina- } \\
\text { Rifampicina }\end{array}$ & $2,30(1,65$ a 3,21$)$ & $1,43,(1,03$ a 2,00$)$ & $\begin{array}{c}\text { Sin diferencias, } \\
\text { (TE 2,3\% de ototoxicidad) }\end{array}$ \\
\hline Quinolona-Rifampicina vs. Doxicicliona-Rifampicina (DR) & $1,40(0,77$ a 2,52$)$ & \multirow[t]{2}{*}{ NS } & $0,37(0,22$ a 0,63$)$ \\
\hline Quinolona-Rifampicina vs Doxixiclina-Estreptomicina & $2,28(1,17$ a 1,46$)$ & & NS \\
\hline \multicolumn{4}{|l|}{ Monoterapia } \\
\hline Con cotrimoxazol & $1,27(0,81$ a 1,99$)$ & & \multirow{4}{*}{ No reportado } \\
\hline Con Tetraciclina & $1,01(0,58$ a 1,77$)$ & No reportado & \\
\hline Ceftriaxona. & $0,25(0,03$ a 2,32$)$ & & \\
\hline Ciprofloxacina vs Terapia combinada & \multicolumn{2}{|c|}{$\begin{array}{c}\text { Suspendidos por desventaja de la } \\
\text { monoterapia }\end{array}$} & \\
\hline Menos de 30 días vs. más de seis semanas & $3,08(1,01$ a 9,38$)$ & No reportado, & \multirow[t]{2}{*}{ Sin diferencias } \\
\hline Estreptomicina-Tetraciclina vs. Gentamicina- Tetraciclina & $2,50(0,56$ a 11$)$ & $0,19(0,01$ a 3,99$)$ & \\
\hline Dos drogas (DR) vs. tres drogas (DR-Aminoglucósido) a & $2,50(1,26$ a 5$)$ & No se observaron & No reportado \\
\hline
\end{tabular}

a Dos ensayos.

\section{Comentario}

La brucelosis es una enfermedad endémica de sintomatología variada y difícil diagnóstico'. La mayoría de los pacientes se curan si reciben el tratamiento durante la primera semana de enfermedad, pero hay discusión sobre cuáles son los mejores esquemas terapéuticos ${ }^{2-4}$. Skalsky y col. hacen hincapié que en menores de ocho años y embarazadas no deben utilizarse tetraciclinas ni quinolonas y describen las recomendaciones resumidas en la tabla 2.

\section{Conclusiones del comentador}

La doxiciclina y los aminoglucósidos son las principales componentes de un doble o triple plan antibiótico ${ }^{5}$. Sin embargo, son necesarios más estudios para establecer la superioridad y seguridad de la triple terapia; debiendo remarcarse que las medidas higiénico-dietéticas constituyen el pilar fundamental en la prevención de la brucelosis.

Tabla 2: recomendaciones para el tratamiento de la brucelosis no complicada (la duración se reporta en semanas).

\begin{tabular}{l|c|c|c} 
& WHO/FAO 1986 & Ioannina $\mathbf{2 0 0 7}$ & \multicolumn{1}{c|}{ Skalsky y col. (revisión actual) } \\
$\begin{array}{l}\text { Primera } \\
\text { IÍnea }\end{array}$ & Doxiciclina más rifampicina (6s) & Doxiciclina (6s) y estreptomicina (2 a 3s) & $\begin{array}{c}\text { Doxiciclina y rifampicina (6s) y gentamicina (2s); } \\
\text { ó doxiciclina (6) más gentamicina (2) }\end{array}$ \\
\hline Alternativa & Tetraciclina (6) más estreptomicina (2 a 3s) & Doxiciclina más rifampicina (6s) & Doxiciclina (6s) más estreptomicina (2s) \\
\hline
\end{tabular}

Dosis orales recomendadas (excepto los aminoglucósidos que son intramusculares incluidas en los ensayos: doxiciclina: 100mg dos veces por día; gentamicina 240mg una vez por día; rifampicina 900mg una vez por día; estreptomicina $1 \mathrm{~g}$ una vez por día; tetraciclina 500mg cuatro veces por día.

Javier Chiarpenello [ Hospital Centenario de Rosario y Centro de Endocrinología de Rosario. jchiarpenello @ hotmail.com ]

Chiarpenello J. Tratamiento de la brucelosis humana. Evid. actual. práct. ambul; 11(4): 105, Jul-Ago.2008. Skalsky K y col. Treatment of human brucellosis: systematic review and meta-analysis of randomised controlled trials. BMJ 2008;336(7646):701-4. PMID: 18321957. Disponible en URL: http://www.bmj.com/cgi/reprint/336/7646/701 (último acceso 30/07/08).

\section{Referencia}

1. Chiarpenello, J y Bessone, M. Brucelosis. Capítulo 263. En: En: "Medicina Familiar y Practica Ambulatoria". Editores Rubinstein A y Terrasa S. Editorial Médica Panamericana, Marzo de 2001. pp 1941-1946.

2. Alp E, y col. Current therapeutic strategy in spinal brucellosis. Int J Infect Dis. 2008 Jun 6.

3. Shen M. Diagnostic and therapeutic challenges of childhood brucellosis in a nonendemic country. Pediatrics. 2008:121(5):e1178-83.

4. Demirtürk N y col. Brucellosis: a retrospective evaluation of 99 cases and review of brucellosis treatment. Trop Doct. 2008 Jan;38(1):59-62.

5. Al-Tawfiq J. Therapeutic options for human brucellosis. Expert Rev Anti Infect Ther. 2008 Feb;6(1):109-20. 